\title{
Angiotensin II Infusion Induces \\ Nephrin Expression Changes and Podocyte Apoptosis
}

\author{
Junya Jia ${ }^{\mathrm{a}}$ Guohua Ding $^{\mathrm{a}}$ Jili Zhu ${ }^{\mathrm{a}}$ Cheng Chen ${ }^{\mathrm{a}}$ Wei Liang ${ }^{\mathrm{a}}$ \\ Nicholas Franki ${ }^{\text {b }}$ Pravin C. Singhal ${ }^{\text {b }}$ \\ ${ }^{a}$ Division of Nephrology, Renmin Hospital, Wuhan University, Wuhan, China; ${ }^{b}$ Department of Medicine, \\ Long Island Jewish Medical Center, New Hyde Park, N.Y., USA
}

\author{
Key Words \\ Angiotensin II - Proteinuria - Nephrin expression • \\ Podocyte $\cdot$ Apoptosis
}

\begin{abstract}
Background/Aim: In in vitro studies, angiotensin (Ang) II has been demonstrated to promote podocyte apoptosis. The present study evaluates the effects of Ang II infusion in rats on podocyte nephrin expression and apoptosis and the molecular mechanisms involved in Ang Il-induced proteinuria and mesangial expansion. Methods: Sprague-Dawley rats were randomly assigned to receive either normal saline or Ang II ( $\left.400 \mathrm{ng} \cdot \mathrm{kg}^{-1} \cdot \mathrm{min}^{-1}\right)$ by means of a mini-osmotic pump for variable time periods. Systolic blood pressure and urinary protein and albumin excretion rate measurements were carried out on days $7,14,21$, and 28 . The animals were sacrificed on days 14 and 28 and evaluated for serum creatinine, renal pathological changes, podocyte apoptosis, renal nephrin mRNA, and protein expression. Results: The Ang II-infused rats developed hypertension and proteinuria. On day 14 , the Ang Il-infused rats showed narrowing of the slit diaphragm, an increase in podocyte nephrin mRNA and protein expression, and alterations in its distribution along the foot processes. On day 28, the Ang II-infused rats demonstrated the presence of apoptotic podocytes and decreased nephrin mRNA and protein expression. There was a negative correla-
\end{abstract}

\begin{tabular}{ll}
\hline KARGER & $\begin{array}{l}\text { (c) 2008 S. Karger AG, Basel } \\
0250-8095 / 08 / 0283-0500 \$ 24.50 / 0\end{array}$ \\
$\begin{array}{l}\text { Fax +4161306 12 34 } \\
\begin{array}{l}\text { E-Mail karger@karger.ch } \\
\text { www.karger.com }\end{array}\end{array}$ & $\begin{array}{l}\text { Accessible online at: } \\
\text { www.karger.com/ajn }\end{array}$
\end{tabular}

tion between nephrin expression and the numbers of apoptotic podocytes $(r=-0.63, p<0.05)$. Conclusion: These results suggest that changes in nephrin expression may play a role in the pathogenesis of Ang II-induced podocyte apoptosis.

Copyright $\odot 2008$ S. Karger AG, Basel

\section{Introduction}

Glomerular visceral epithelial cells, also called podocytes, are located outside of the glomerular basement membrane and maintain its structural and functional integrity. Recent studies have demonstrated that podocytes play an important role in the pathogenesis and progression of chronic kidney diseases [1-4]. Angiotensin (Ang) II is one of the major effector molecules of the renin-angiotensin system (RAS). Its role in the development of glomerular sclerosis that progresses to end-stage renal disease is increasingly recognized [5-8]. Its infusion has been shown to accelerate renal fibrosis in a mouse model of HIV-1-associated nephropathy [9]. On the other hand, its blockade of action or production has also been shown

This work was presented at the 39th Annual Renal Week Meeting of the American Society of Nephrology, San Diego, Calif., USA, November 2006.
Dr. Guohua Ding

Division of Nephrology, Renmin Hospital, Wuhan University

238 Jiefang Road

Wuhan 430060 (China)

Tel. +86 278804 1911, Fax +86 278804 229, E-Mail GXD101@yahoo.com 
to result in diminution of proteinuria as well as slowing down of the progression of renal fibrosis in mice transgenic for HIV-1 [10, 11]. Similar results have been demonstrated in rat models of diabetes and hypertension [12, 13]. Moreover, the beneficial effects of Ang II inhibition have been reported in a variety of human renal diseases [14-16]. Therefore, Ang I-converting enzyme inhibitors and Ang II receptor blockers are the mainstays of therapy utilized to slow down the progression of proteinuric renal diseases in humans [14-16].

Podocytes express Ang II receptors and have been shown to be target cells for its action [17]. Hoffmann et al. [4] demonstrated that Ang II type 1 receptor overexpression in podocytes inflicted marked structural changes and nephron loss in transgenic rats. These rats developed proteinuria and progressive glomerulosclerosis [4]. Thus, it appears that not only excessive production of Ang II but also its enhanced action, despite normal levels, is equally effective in inflicting the target organ damage.

Nephrin, a transmembrane protein of the slit diaphragm, has been demonstrated to play a critical role in the development of proteinuric glomerular diseases [1-3]. Ang II infusion has been shown to induce alterations in nephrin mRNA expression in podocytes [18]. In addition, Ang II promoted apoptosis of cultured glomerular podocytes in in vitro studies [19]. We hypothesize that Ang II infusion has a potential to induce podocyte apoptosis in association with an alteration of nephrin expression.

Ang II is a well-known effector molecule for the development of secondary hypertension in patients with renal artery stenosis [20]. Hypertension can also be induced and maintained with continuous infusion of Ang II through a mini-osmotic pump in experimental rat models to mimic different forms of essential hypertension in humans $[21,22]$. Using such models, structural and functional changes have been demonstrated to be accompanied by increments in circulating and intrarenal Ang II levels [21, 22]. To evaluate the effects of Ang II infusion on the expression of nephrin and podocyte apoptosis, we used an Ang II-infused hypertensive rat model.

\section{Materials and Methods}

\section{Animals and Experimental Design}

Thirty-six male Sprague-Dawley rats (Animal Laboratories of the Wuhan University, Wuhan, China) weighing between 180 and $220 \mathrm{~g}$ were housed in cages and maintained in a temperature-controlled room with a 12:12-hour light-dark cycle, with free access to tap water and standard rat chow for 2 or 4 weeks. For celiac mini-pump implantation (Alzet model 2002 or 2004; Alza, Mountain View, Calif., USA), rats were selected at random to be subjected to either human Ang II infusion (Sigma Chemical, St. Louis, Mo., USA) at $400 \mathrm{ng} \cdot \mathrm{kg}^{-1} \cdot \mathrm{min}^{-1}$ for 14 or 28 days $(\mathrm{n}=6)$ or normal saline infusion for 14 or 28 days $(n=6)$ or to be used as normal controls ( 14 or 28 days, $\mathrm{n}=6$ ).

\section{Systolic Blood Pressure Measurement}

The systolic blood pressure was monitored by tail cuff plethysmography (China-Japan Friendship Hospital, Beijing, China) in conscious, trained, and preheated rats on days $0,7,14,21$, and 28.

\section{Sample Collection and Preparation}

Urine samples were collected throughout a 24-hour period to assay for proteinuria and albuminuria on days $0,7,14,21$, and 28 . Blood and kidney samples were harvested on days 14 and 28 . Trunk blood was collected into prechilled tubes, then centrifuged at $4,000 \mathrm{rpm}$ for $30 \mathrm{~min}$ at $4^{\circ} \mathrm{C}$. Plasma fractions were removed and assayed for creatinine.

After decapsulation, the kidneys were washed with ice-cooled saline, blotted dry, and weighed. The left kidney was cross-sectioned and fixed in $10 \%$ formalin in phosphate-buffered saline (PBS, pH 7.2) for histochemical and TUNEL studies (see below). The right renal cortex was separated and divided into three parts: the first one was embedded in Tissue-Tek OCT compound and snap frozen at $-80^{\circ} \mathrm{C}$ without prior fixation for immunofluorescence staining, the second one was snap frozen in liquid nitrogen for Western blot and RT-PCR analyses, and the third one was fixed with $2.5 \%$ glutaraldehyde or with phosphate-buffered $3.5 \%$ paraformaldehyde plus $0.02 \%$ glutaraldehyde for transmission electron microscopic and immune transmission electron microscopic analyses, respectively.

\section{Immunofluorescence Microscopic Analysis}

Frozen sections $(6 \mu \mathrm{m})$ were transferred to slides, washed with cold PBS, and processed as follows: Sections were fixed with chilled acetone for $10 \mathrm{~min}$ at $-20^{\circ} \mathrm{C}$ and incubated with $0.1 \%$ Triton $\mathrm{X}-100$ in PBS at $37^{\circ} \mathrm{C}$ for $15 \mathrm{~min}$. After washing with PBS, the sections were then blocked with $1 \%$ bovine serum albumin for 30 min and stained with goat anti-human nephrin (1:50; Santa Cruz Biotechnology, Santa Cruz, Calif., USA) for $12 \mathrm{~h}$ at $4{ }^{\circ} \mathrm{C}$, followed by rhodamine-conjugated rabbit anti-goat IgG (1:100; Zhongshan, Beijing, China). As a control, some sections were stained with nonassociated antibody instead of anti-nephrin. The sections were examined by fluorescence microscopy (BX51 microscope; Olympus, Tokyo, Japan). The images were captured with a spot charge-coupled device camera (Olympus). All exposure settings were kept constant for each primary antibody.

\section{TUNEL Assay}

DNA fragmentations in apoptotic cells were detected using the TUNEL assay. After paraffin dewaxing, sections $(5 \mu \mathrm{m})$ were incubated with $3 \% \mathrm{H}_{2} \mathrm{O}_{2}$ for $30 \mathrm{~min}$ followed by $0.1 \%$ Triton X-100 in PBS for $15 \mathrm{~min}$ at room temperature. Sections were washed and exposed to TdT buffer for $5 \mathrm{~min}$ and incubated in a moist chamber with a mixture of TdT and digoxigenin-11-dUTP in TdT buffer (R\&D Systems, Minneapolis, Minn., USA) for $1 \mathrm{~h}$ at room temperature. They were then washed in PBS for $15 \mathrm{~min}$. The sections were incubated for $30 \mathrm{~min}$ with streptavidin-biotin-peroxidase- 

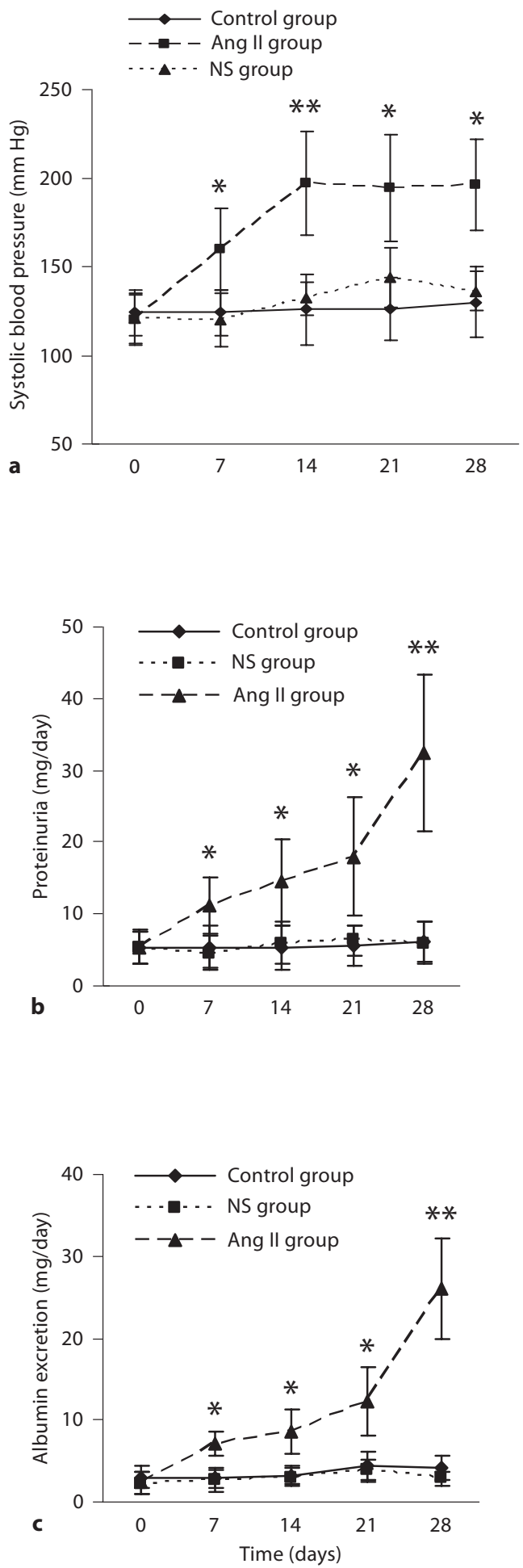

Fig. 1. Ang II-infused rats developed hypertension (a), proteinuria (b), and albuminuria (c). NS $=$ Normal saline. ${ }^{*} \mathrm{p}<0.05$; ${ }^{* *} \mathrm{p}<0.01$ vs. control group. conjugated anti-digoxigenin-11-dUTP antibody, and antibodybinding sites were visualized using diaminobenzidine. The slides were counterstained with hematoxylin. Negative controls included the omission of TdT; positive controls included the pretreatment of sections with $0.1 \mathrm{U} / \mu$ l deoxynuclease- 1 before TdT staining. Apoptotic podocytes from single cross-sections through the glomerulus were counted using the Weibel-Gomez method.

\section{Transmission Electron Microscopic Analysis}

Paraformaldehyde-glutaraldehyde-fixed 1- $\mathrm{mm}^{3}$ blocks of renal cortices were postfixed with $1 \%$ osmium in $0.1 \mathrm{M}$ cacodylate buffer for $1 \mathrm{~h}$, dehydrated in graded ethanols, embedded in Epon, sectioned, stained with uranyl acetate and lead citrate, and examined and photographed with a Hitachi H600 transmission electron microscope (Hitachi, Tokyo, Japan).

\section{Immune Electron Microscopic Analysis}

Paraformaldehyde-glutaraldehyde-fixed, $1-\mathrm{mm}^{3}$ blocks of renal cortex were washed with PBS, dehydrated in graded ethanols, and embedded in Epon. Ultrathin sections were transferred to nickel grids and then blocked with $1 \%$ bovine serum albumin and $1 \%$ normal goat serum in PBS. Sections were incubated with the primary polyclonal goat anti-human nephrin antibody against the intracellular domain of nephrin (1:50; Santa Cruz Biotechnology) and then with the secondary gold-conjugated $(10 \mathrm{~nm})$ rabbit anti-goat secondary antibody (1:100; R\&D Systems). The sections were postfixed with $1 \%$ glutaraldehyde, contrasted with uranyl acetate and lead citrate, then observed under an electron microscope and photographed for detailed analysis.

\section{Reverse Transcription-Polymerase Chain Reaction}

Total RNA was extracted with TRIzol reagent (Promega, Madison, Wisc., USA). Total RNA (5 $\mu \mathrm{g})$ extracted from renal cortex was used to synthesize cDNA and served as a template for amplification of nephrin and for glyceraldehyde-3-phosphate dehydrogenase (GAPDH) as an endogenous standard. The forward and reverse primer sequences (Shenggong, Shanghai, China) were for nephrin 5'-AGCCTCTTGACCATCGCTAA and 5'-CCCAGTCAGCGTGAAGGTAG, respectively, and for GAPDH 5'-ACAGAGTACTTGCGCTCAGGAG and 5'-GTCACCCACACTGTGCCCATC, respectively. The PCR products were 302 and $542 \mathrm{bp}$, respectively. The amplification was performed under the following conditions: $94^{\circ} \mathrm{C}$ for $45 \mathrm{~s}$ (denaturation), $56^{\circ} \mathrm{C}$ for $45 \mathrm{~s}$ (annealing), and $72^{\circ} \mathrm{C}$ for $1 \mathrm{~min}$ (extension), with 35 cycles at $72^{\circ} \mathrm{C}$ for $10 \mathrm{~min}$ for final extension. PCR products were analyzed by electrophoresis and the intensity of the bands by means of AlphaEase FC image software (Alpha Innotech, San Leandro, Calif., USA).

\section{Western Blotting Studies}

The renal cortex sample was homogenized on ice and the proteins extracted using a lysis buffer, centrifuged at $12,000 \mathrm{~g}$ for 15 min at $4^{\circ} \mathrm{C}$, and the resultant supernatant was collected. The protein concentration of the samples was determined with a BCA protein assay kit (Pierce Biotechnology, Rockford, Ill., USA). Western blotting analysis was performed as follows: Samples were separated on an $8 \%$ polyacrylamide gel and then semidry blotted on a PVDF membrane. The blotted membrane was incubated for $2 \mathrm{~h}$ at room temperature in blocking solution (5\% blocking agent in $1 \%$ defatted milk) in Tris-buffered saline (TBS) with $0.02 \%$ Tween $20(\mathrm{~T})$ and then washed three times with washing solution. 
Fig. 2. a Normal saline infusion on day 28. Ang II induced mesangial proliferation on day 14 (b) and segmental sclerosis on day 28 (c). PAS. $\times 400$
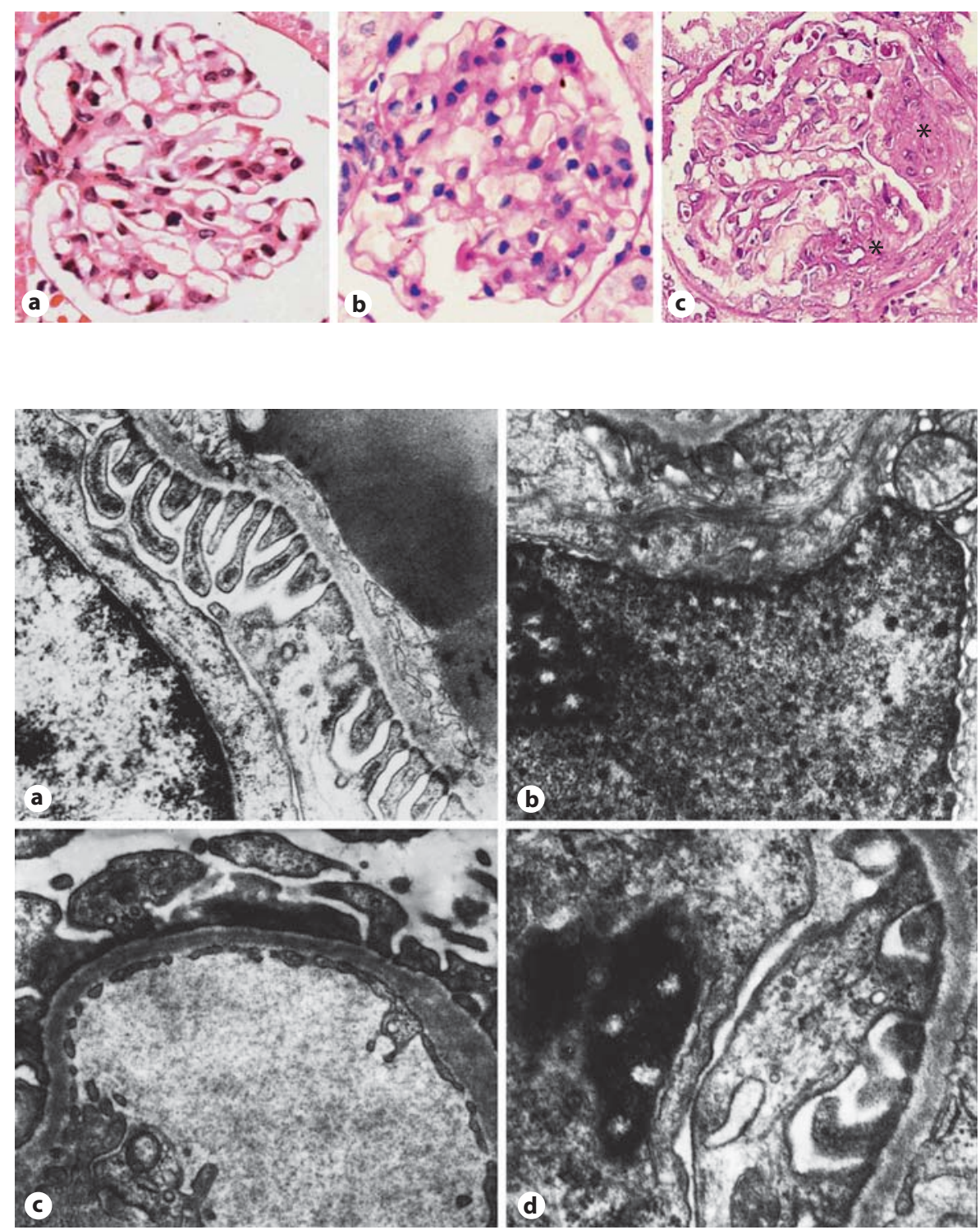

Fig. 3. a Normal control. Ang II induced narrowing of foot processes, disordered microfilament bundle arrangement, and increase of nuclear euchromatin on day 14 (b) and broadening and segmental fusion of foot processes (c) and podocyte apoptosis $($ d) on day 28 . TEM. $\times 20,000$.

The blocked membrane was incubated overnight at $4^{\circ} \mathrm{C}$ with primary antibodies (1:50; Santa Cruz Biotechnology) in the TBS-T solution containing $1 \%$ bovine serum albumin and then with rabbit anti-goat secondary antibody (1:100; Zhongshan). The detection was performed according to the manufacturer's instructions using the ECL kit (Santa Cruz Biotechnology). The intensity of the bands was analyzed using Alpha Ease FC image software.

\section{Statistical Analysis}

Statistical analysis was performed using SPSS software version 10.0 (SPSS, Chicago, Ill., USA). Values are presented as mean \pm $\mathrm{SD}$ or as mean \pm SEM. Testing between two groups was performed by Student's t test, and one-way analysis of variance was used for comparing three groups. Correlations were assessed by the Spearman rank correlation coeffiencent (r). p $<0.05$ was considered statistically significant.

Nephrin Expression and Podocyte Apoptosis

\section{Results}

\section{Blood Pressure, Proteinuria, and Serum Creatinine} Profiles

The temporal changes in systolic blood pressure of rats in each group are shown in figure 1a. The systolic blood pressure was identical among the groups on day 0 and was not changed during 28 days in control and normal saline-infused rats. Ang II-infused rats developed hypertension (204 $\pm 10 \mathrm{~mm} \mathrm{Hg}$ on day 28). Proteinuria and albuminuria in Ang II-infused rats were detectable and represented the onset of glomerular injury (fig. 1b, c). Proteinuria was elevated on day 7 and was $>30 \mathrm{mg} / \mathrm{l}$ on day 28. However, none of the Ang II-infused rats had 
Fig. 4. Normal control (a) and Ang II infusion on day 14 (b). c Ang II induced podocyte apoptosis (2.7 \pm 0.8/glomerular cross-section) on day 28 . TUNEL. $\times 400$.

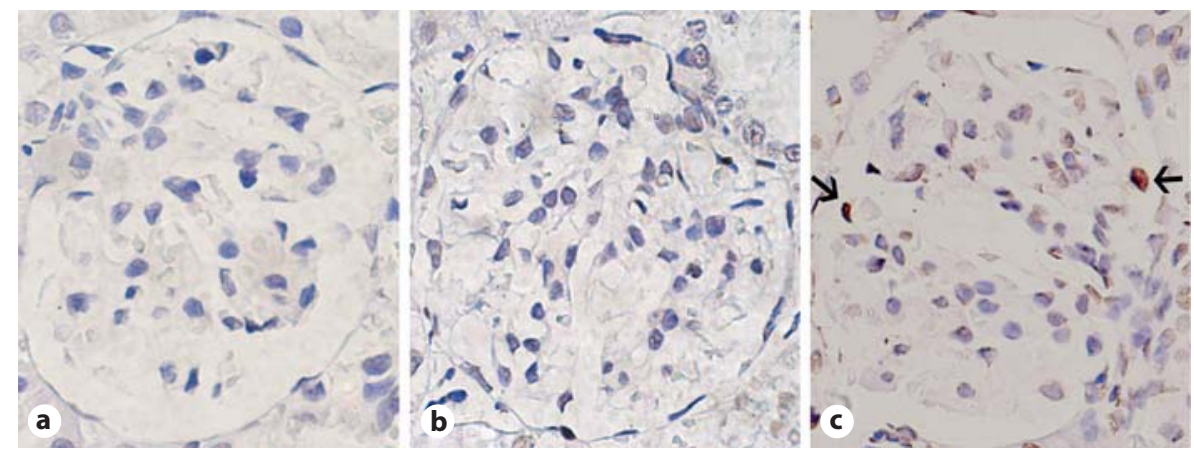

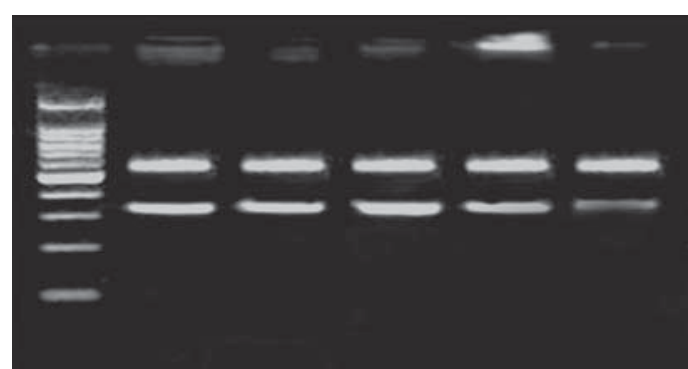

GAPDH Nephrin

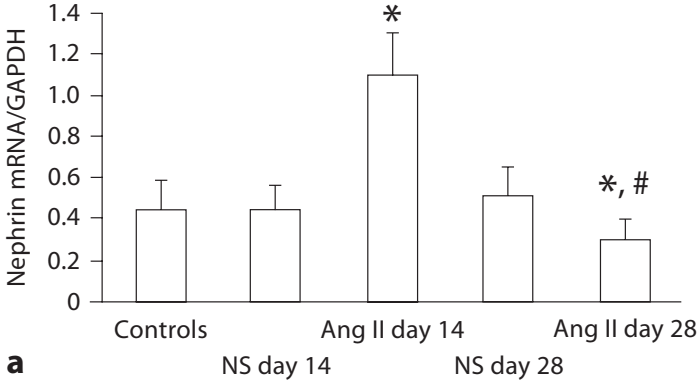

significant changes in plasma creatinine levels on day 28.

\section{Histological and Electron Microscopic Changes}

The histological findings in the normal saline group on day 28 and in the Ang II group on days 14 and 28 are shown in figure 2. Normal saline-infused rats showed normal glomeruli on day 28 (fig. 2a). By contrast, mild mesangial proliferation was exhibited in Ang II-infused rats on day 14 (fig. $2 \mathrm{~b}$ ) and became more prominent on day 28. Segmental sclerotic glomeruli were occasionally observed in Ang II-infused animals on day 28 (fig. 2c).

Electron microscopic analysis of glomeruli in the Ang II-infused rats on day 14 revealed podocytes with foot

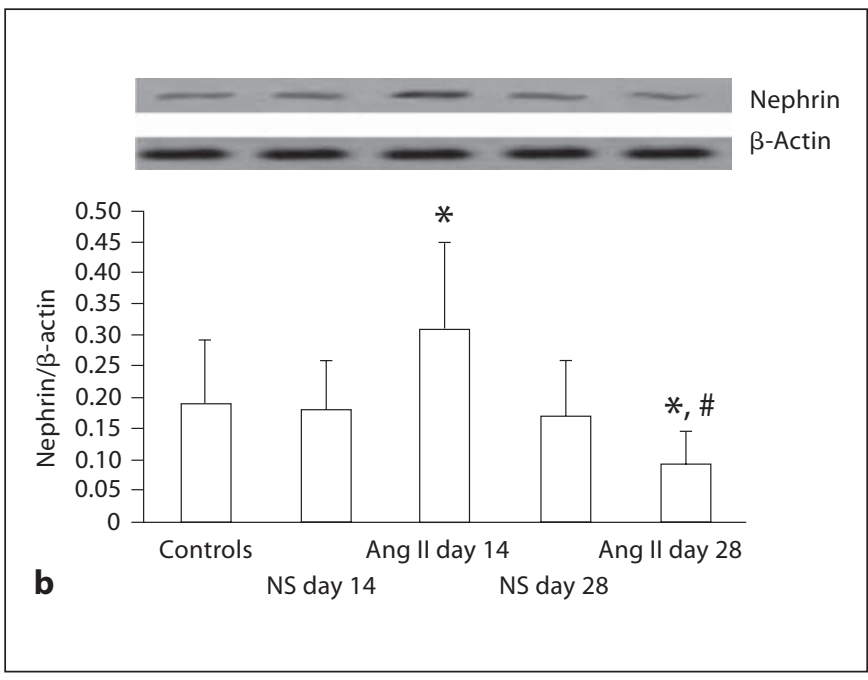

Fig. 5. Ang II-induced expression of nephrin mRNA (a) and nephrin (b). Both increased on day 14 and then markedly decreased on day 28. NS = Normal saline. ${ }^{*} \mathrm{p}<0.05$ vs. control group; ${ }^{\#} \mathrm{p}<0.05$ vs. Ang II group on day 14 .

processes in closer apposition, narrowed slit diaphragms, indiscriminate microfilament bundle, and enriched nuclear euchromatin (fig. 3 b). On day 28 , the glomeruli developed the broadening and segmental fusion of foot processes, devoid of slit diaphragms, accompanied by derangement of actin filaments (fig. 3c). Podocyte apoptosis was detected on day 28 (fig. $3 \mathrm{~d}$ ).

\section{Podocyte Apoptosis Detected by TUNEL Assay}

There were no apoptotic podocytes in the rats infused with normal saline. By contrast, a significant increase in apoptotic podocytes per glomerular section was detected on day 28 in Ang II-infused rats, but not on day 14 (fig. 4). 
Fig. 6. The distribution of the nephrin expression changed from a linear pattern in a control rat (a) to a granular pattern (b) after Ang II infusion for 14 days. The nephrin expression decreased by day 28 (c). $\times 400$.
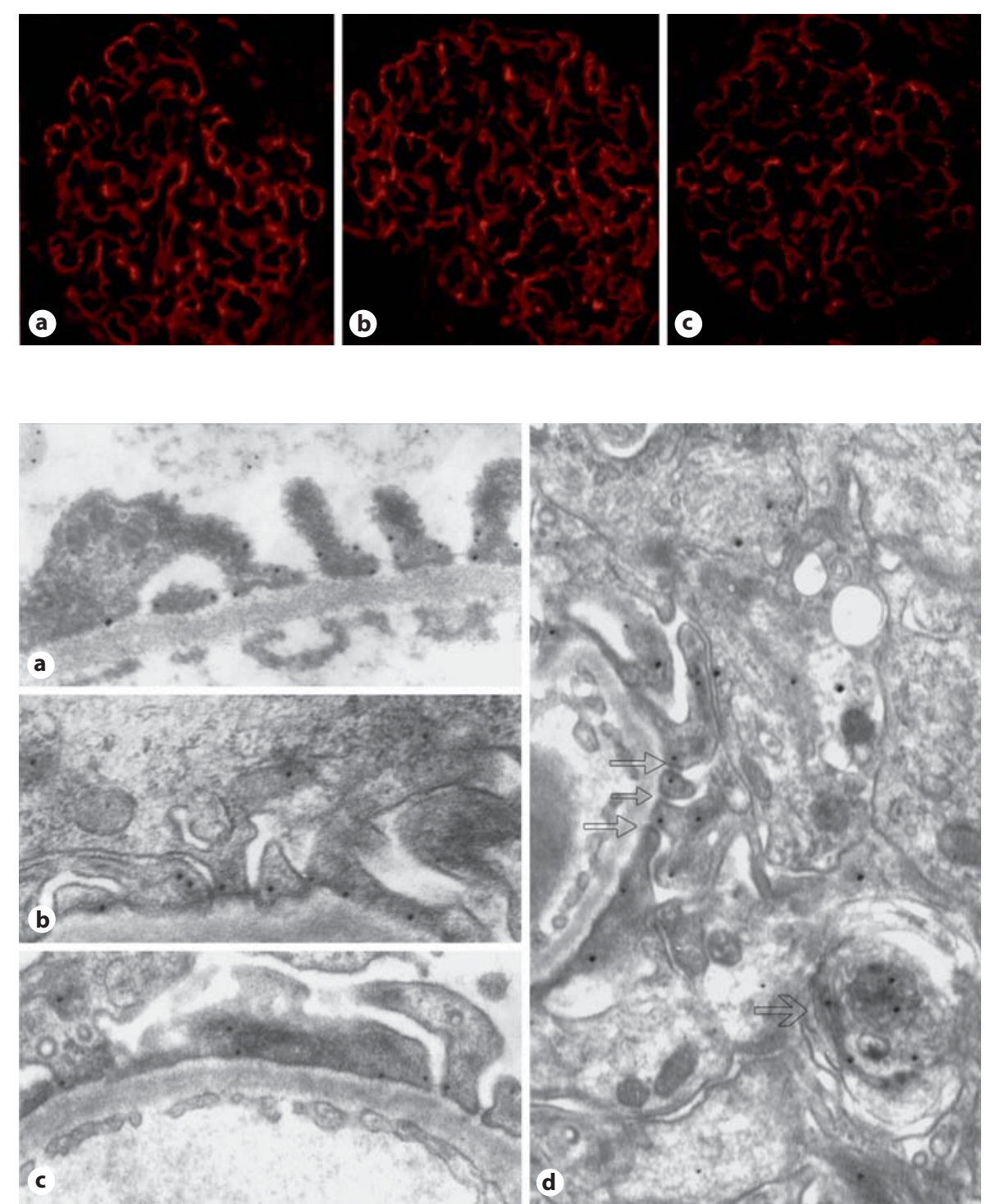

Fig. 7. a Normal control. Immunogold labeling for nephrin on the slit diaphragm (b) but increased in cytoplasm and vesicles (d) on day 14. c The total number of gold particles decreased on day 28 . a-c $\times 30,000 . \mathbf{d} \times 25,000$.

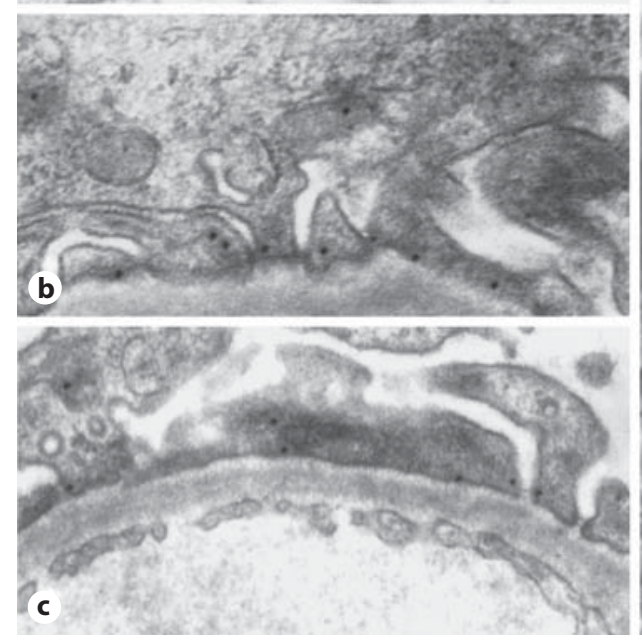

d

Nephrin mRNA and Protein Expression

Nephrin mRNA and protein levels were elevated on day 14 in the renal cortex of Ang II-infused rats, as shown by RT-PCR and Western blotting (fig. 5). To determine the changes of the nephrin distribution pattern, we performed immunofluorescence labeling of glomerular sections. The nephrin expression was distributed linearly along the glomerular basement membrane in control and normal saline-infused rats, whereas the pattern was changed to a dispersed and granular distribution on day 14 in Ang II-infused rats (fig. 6a, b). By contrast, nephrin mRNA and protein expression decreased markedly on day 28 in Ang II-infused rats (fig. 5, 6c). In order to de- termine the localization of nephrin on the podocyte slit diaphragms in detail, we performed immunogold labeling of nephrin on ultrathin sections. In glomeruli of control and normal saline-infused rats, the gold particles were located on the slit diaphragms or the foot process surfaces (fig. 7a). In Ang II-infused rats, however, the numbers of gold particles on the slit diaphragms were decreased markedly, and most of them were located in the cytoplasm and vesicles on day 14 (fig. 7b, d). In accordance with the findings from immunofluorescence staining, the total number of gold particles located either on slit diaphragms or in podocyte cytoplasm decreased dramatically on day 28 (fig. 7c). 
Podocyte Apoptosis in Association with Decreased Nephrin Levels in Ang II-Infused Rats on Day 28

A highly significant negative correlation was found between nephrin expression and the number of apoptotic podocytes in Ang II-infused rats on day $28(\mathrm{r}=-0.63$, $\mathrm{p}<0.01)$.

\section{Discussion}

In the present study, Ang II-infused rats developed hypertension, narrowing of slit diaphragm, and proteinuia. Interestingly, Ang II infusion resulted in a bimodal effect on podocyte nephrin expression. Podocyte nephrin expression increased on day 14 but decreased on day 28 of Ang II infusion. There was a negative correlation between the occurrence of podocyte apoptosis and nephrin expression in Ang II-infused rats. These findings indicate that there may be a relationship between the occurrence of podocyte apoptosis and alterations in nephrin expression.

Ang II is one of the major effector molecules of the RAS. In the kidney, it acts as a circulating hormone as well as a paracrine/autocrine cytokine to modulate renal function. Activation of the local renal RAS exacerbates the progression of chronic kidney disease, and inhibition of the RAS activity markedly retards proteinuria and progression to end-stage renal disease $[1,2]$. Podocytes express both Ang II receptors, type 1 and type 2 [17], thus, playing an important role in the progression of chronic renal disease due to the activation of the local renal RAS [23-26]. Overexpression of Ang II type 1 receptor in rats has been demonstrated to induce proteinuria, nephron loss, and development of glomerulosclerosis [4]. The present study also shows that Ang II-infused rats developed hypertension and proteinuria. Ang II infusion induced mild to moderate mesangial proliferation in glomeruli and caused structural damages in podocytes. Ang II might induce proteinuria through two mechanisms: by increment of glomerular capillary pressure or - through its direct cellular effect independent of hemodynamics - direct podocyte injury.

Podocytes have been suggested to be the key cells responsible for development of proteinuric renal diseases [26]. Nephrin, a structural molecule of podocytes, plays a key role in the pathogenesis of proteinuria [2-4, 24-26]. Doublier et al. [27] have shown that Ang II infusion caused changes in nephrin mRNA expression. In the present study, we have demonstrated that the nephrin expression pattern was different on day 14 versus day 28 in
Ang II-infused animals. At present, the molecular mechanism involved in this bimodal effect of Ang II is not clear.

Nephrin has been considered as an adhesion molecule in cell-cell interaction and as a signal-transducing receptor through tyrosine phosphorylation at its $\mathrm{C}$ terminus $[28,29]$. Its loss or endocytosis may reduce tyrosine phosphorylation and terminate its signaling. It promotes cellcell adhesion through homophilic interactions, and thus its redistribution in podocytes may lead to proteinuria $[30,31]$. We speculate that, in the present study, the alteration of nephrin expression may be a reaction of podocytes to mechanical stress, induced by enhanced Ang II activity.

Podocyte depletion has been considered an important phenomenon in proteinuric glomerulopathy for many years, and is now considered to play a central role in the progression of renal diseases $[1-3,24]$. We have recently demonstrated that Ang II induces apoptosis of cultured rat podocytes [19]. In Ang II-infused rats, on day 28, podocyte apoptosis was detected by electron microscopy as well as TUNEL assay. Proteinuria increased markedly by day 14 and day 28 . We presume that podocyte apoptosis contributes to the occurrence of proteinuria. Interestingly, a highly significant negative correlation was found between nephrin expression and apoptotic podocytes. Our data show that Ang II infusion induces nephrin derangement, or even its decrement, and the latter might play a role in the induction of podocyte apoptosis.

In conclusion, these results suggest that Ang II-infused rats develop hypertension, proteinuria, alteration in nephrin expression, and podocyte apoptosis. The changes in nephrin expression may play a role in the pathogenesis of Ang II-induced podocyte apoptosis.

\section{Acknowledgments}

G. Ding was supported by National Nature Science Foundation Grant of China No. 30370656 and P.C. Singhal by grant No. 10RO1DA12111 from the National Institutes of Health (Bethesda, Md., USA). 


\section{References}

${ }_{1}$ Benigni A, Gagliardini E, Remuzzi G: Changes in glomerular perm-selectivity induced by angiotensin II imply podocyte dysfunction and slit diaphragm protein rearrangement. Semin Nephrol 2004;24: 131-140.

$\checkmark 2$ Macconi D, Bonomelli M, Benigni A, et al: Pathophysiologic implications of reduced podocyte number in a rat model of progressive glomerular injury. Am J Pathol 2006; 168:42-54.

3 Durvasula RV, Shankland SJ: Podocyte injury and targeting therapy: an update. Curr Opin Nephrol Hypertens 2006;15:1-7.

4 Hoffmann S, Podlich D, Hähnel B, et al: Angiotensin II type 1 receptor overexpression in podocytes induces glomerulosclerosis in transgenic rats. J Am Soc Nephrol 2004; 15 1475-1487.

5 Hilgers KF, Mann JF: Role of angiotensin II in glomerular injury: lessons from experimental and clinical studies. Kidney Blood Press Res 1996;19:254-262.

6 Mahmood J, Khan F, Okada S, et al: Local delivery of angiotensin receptor blocker into the kidney ameliorates progression of experimental glomerulonephritis. Kidney Int 2006;70:1591-1598.

7 Nakamura T, Obata J, Kimura H, et al: Blocking angiotensin II ameliorates proteinuria and glomerular lesions in progressive mesangioproliferative glomerulonephritis. Kidney Int 1999;55:877-889.

$\checkmark 8$ Zoja C, Abbate M, Corna D, et al: Pharmacologic control of angiotensin II ameliorates renal disease while reducing renal TGF-beta in experimental mesangioproliferative glomerulonephritis. Am J Kidney Dis 1998;31: 453-463.

9 Ideura H, Hiromura K, Hiramatsu N, et al: Continuous angiotensin II (AII) infusion exaggerates podocyte injury in mouse model of HIV-associated nephropathy (HIVAN). J Am Soc Nephrol 2006;17:72A.
10 Hiramatsu N, Hiromura K, Shigehara T, et al: Angiotensin II type 1 receptor blockade inhibits the development and progression of $\mathrm{HIV}$-associated nephropathy in a mouse model. J Am Soc Nephrol 2007;18:515-527.

11 Bird JE, Durham SK, Giancarli MR, et al: Captopril prevents nephropathy in HIVtransgenic mice. J Am Soc Nephrol 1998;9: 1441-1447.

12 Rosenthal T, Erlich Y, Rosenmann E, et al: Effects of enalapril, losartan, and verapamil on blood pressure and glucose metabolism in the Cohen-Rosenthal diabetic hypertensive rat. Hypertension 1997;29:1260-1264.

13 Gross ML, Ritz E, Schoof A, et al: Renal damage in the SHR/N-cp type 2 diabetes model: comparison of an angiotensin-converting enzyme inhibitor and endothelin receptor blocker. Lab Invest 2003;83:1267-1277.

14 de Zeeuw D, Remuzzi G, Parving HH, et al: Proteinuria, a target for renoprotection in patients with type 2 diabetic nephropathy: lessons from RENAAL. Kidney Int 2004;65: 2309-2320.

15 Lewis EJ, Lewis JB: Treatment of diabetic nephropathy with angiotensin II receptor antagonist. Clin Exp Nephrol 2003;7:1-8.

16 Andersen S, Tarnow L, Rossing P, et al: Renoprotective effects of angiotensin II receptor blockade in type 1 diabetic patients with diabetic nephropathy. Kidney Int 2000;57:601606.

17 Sharma M, Sharma R, Greene AS, et al: Documentation of angiotensin II receptors in glomerular epithelial cells. Am J Physiol Renal Physiol 1998;274(3 Pt 2):F623-F627.

18 Zhu J, Ding G, Chen C, et al: Angiotensin II infusion causes proteinuria and changes in nephrin expression. J Am Soc Nephrol 2004; 15:486A

19 Ding G, Reddy K, Kapasi A, et al: Angiotensin II induces apoptosis in rat glomerular epithelial cells. Am J Physiol Renal Physiol 2002;283:F173-F180.

20 Melaragno MG, Fink GD: Enhanced slow pressor effect of angiotensin II in two-kidney, one-clip rats. Hypertension 1995;25: 288-293.

21 Reckelhoff JF, Romero JC: Role of oxidative stress in angiotensin-induced hypertension. Am J Physiol Regul Integr Comp Physiol 2003;284:R893-R912.
22 Navar LG, Harrison-Bernard LM: Intrarenal angiotensin II augmentation in angiotensin II dependent hypertension. Hypertens Res 2000;23:291-301

23 Remuzzi G, Perico N, Macia M, et al: The role of renin-angiotensin-aldosterone system in the progression of chronic kidney disease. Kidney Int Suppl 2005;99:S57-S65.

24 Foster RR, Saleem MA, Mathieson PW, et al: Vascular endothelial growth factor and nephrin interact and reduce apoptosis in human podocytes. Am J Physiol Renal Physiol 2005;288:F48-F57.

25 Wolf G, Chen S, Ziyadeh FN: From the periphery of the glomerular capillary wall toward the center of disease: podocyte injury comes of age in diabetic nephropathy. Diabetes 2005;54:1626-1634.

26 Wharram B, Goyal M, Wiggins JE, et al: Podocyte depletion causes glomerulosclerosis: diphtheria toxin-induced podocyte depletion in rats expressing human diphtheria toxin receptor transgene. J Am Soc Nephrol 2005; 16:2941-2952.

27 Doublier S, Salvidio G, Lupia E, et al: Nephrin expression is reduced in human diabetic nephropathy: evidence for a distinct role for glycated albumin and angiotensin II. Diabetes 2003;52:1023-1030.

28 Verma R, Wharram B, Kovari I, et al: Fyn binds to and phosphorylates the kidney slit diaphragm component Nephrin. J Biol Chem 2003;278:20716-20723.

29 Huber TB, Hartleben B, Kim J, et al: Nephrin and CD2AP associate with phosphoinositide 3-OH kinase and stimulate AKT-dependent signaling. Mol Cell Biol 2003;23:4917-4928.

30 Doublier S, Ruotsalainen V, Salvidio G, et al: Nephrin redistribution on podocytes is a potential mechanism for proteinuria in patients with primary acquired nephrotic syndrome. Am J Pathol 2001;158:1723-1731.

31 Khoshnoodi J, Sigmundsson K, Öfverstedt L, et al: Nephrin promotes cell-cell adhesion through homophilic interactions. Am J Pathol 2003; 163:2337-2346. 